\title{
SISTEMAS CONSTRUCTIVOS INDUSTRIALIZADOS
}

\author{
(INDUSTRIALIZED CONSTRUCTIVE SYSTEMS)
}

\author{
Alfonso del Águila García, Dr. Arquitecto \\ Catedrático de "Construcciónes Arquitectónicas" de la ETSAM. Asesor de la Fundación Cultural COAM
}

ESPAÑA

Fecha de recepción: $31-\mathrm{X}-96$

$193-26$

\section{RESUMEN \\ El presente articulo resume lo tratado en tres jornadas de Sistemas Constructivos Innovadores, relativos a diversos temas de Construcción Industrializada.}

En la primera parte se trata el tema de los paneles de hormigón aplicados a la edificación, principalmente a viviendas. Se da una sintesis histórica y esquemática de conceptos generales, para terminar con una descripción del Sistema Constructivo INDAGSA que están realizando numerosas promociones en Madrid.

La segunda parte, desarrolla el tema de la construcción con módulos tridimensionales, comenzando por unas consideraciones generales que abarcan el concepto de módulo tridimensional, su clasificación, aspectos históricos y campos de aplicación. Se sigue con una descripción del Sistema SET HOME, de módulos tridimensionales de hormigón, dedicado a la industrialización de servicios e instalaciones en vivienda social. Termina esta parte con una descripción del Sistema francés TRANSLOKO, de grandes módulos tridimensionales ligeros, que ha realizado últimamente un edificio para laboratorios en Madrid.

La tercera y última parte se dedica a un tema novedoso dentro de la construcción de interiores, que es la "solera seca", describiéndose detalladamente el enfoque que hace PLADUR sobre el particular.

\section{SUMMARY}

The present article summarizes the main points discussed in three Innovator Buildings Systems sessions, which are about different subjects of Industrialized Building.

The first part is about the topic of concrete panels, applied to building flats basically. It is given a historical summary and the general concepts, ending with "INDAGSA BUILDING SYSTEM", wich is running several promotions with in Madrid.

In the second part it is developed the subject of building with boxes, beginning with general points which are extended to the concept of box, its clasification, its historical aspects and its applications. It is continues with a description of the "SET HOME SYSTEM" of boxes of concrete, which is dedicated to industrialization of social housing services and equipments. This part ends with the french system "TRANSLOKO" of big light boxes. Lately, it has made a building for laboratories in Madrid.

The last part is dedicated to a new subject in Interior Building, which is the Dry Floor, being detailedly described the "PLADUR" point of view about this. 


\section{EDIFICAR SIN LADRILLOS}

\section{CONCEPTOS GENERALES DE LOS SISTEMAS DE PANELES DE HORMIGÓN}

\subsection{Síntesis histórica}

Los sistemas actuales de paneles tienen sus precedentes en experimentaciones realizadas con diversas clases de hormigón desde mediados del siglo XIX. Nombres tales como los de Godwin, Coignet, Edison, Mopin y Perret, son representación de otras tantas experiencias en el desarrollo de los prefabricados de hormigón para la edificación. Sin duda, ocupa un lugar preferente en esta evolución histórica Le Corbusier que, habiendo trabajado en el estudio de Perret, llevó a cabo realizaciones tan famosas como la inicial "casa Loucheur" o, más posteriormente, con sus "Unidades de Habitación".

No obstante todos estos precedentes, serios y documentados, hubo que esperar que finalizase la II Guerra Mundial para que la necesidad de viviendas, dada la enorme destrucción sufrida en Europa y la escasez de mano de obra, debida a las víctimas de la contienda, incidiesen en la búsqueda de sistemas industrializados que satisficieran lo más rápidamente posible la demanda. Que la solución se encontrase en los procedimientos de paneles prefabricados de hormigón no puede, por tanto, deberse a una casualidad, sino a una cierta preparación tecnológica latente. Francia, Gran Bretaña, Alemania, Italia y Países Nórdicos se convierten en focos de realizaciones y de exportación mundial de sus sistemas, en lo que siempre ha sido líder el país galo.

Los antiguos países socialistas se adscribieron también a estas tecnologías, con mayor o menor fortuna de calidad, llegando a abordar con dichos sistemas la mayoría de los programas de construcción de viviendas, realizados dentro de su planificación económica centralizada.

En España se hicieron algunos balbuceos en los años cuarenta, como el sistema Copanel, en el que intervino el Pf. Cámara Niño, pero la construcción masiva de viviendas se llevó a cabo por sistemas tradicionales. Es verdad que existieron, a principios de los sesenta, una serie de tentativas de realizaciones con grandes paneles, según sistemas franceses que tuvieron poco éxito. Hubo que esperar a comienzos de los setenta para la realización de experiencias más serias en el terreno de la construcción industrializada en España, constituyéndose fábricas importantes para la producción de grandes paneles, según sistemas franceses y daneses, principalmente, existiendo, también, uno ruso y varios de origen español. Con todos ellos se realizaron conjuntos de viviendas, siguiendo el porcentaje que se reservaba entonces en los programas VPO para experiencias industrializadas. La depresión económica de principios de los setenta y sus negativos efectos en el Sector de la construcción, produjo una sucesiva paralización y cierre de la mayoría de estas factorías

En la actualidad se observa un resurgir, sobre todo, en la zona Centro.

\subsection{Concepto y características de los paneles prefabri- cados de hormigón}

Se conoce por panel prefabricado de hormigón a un elemento-lámina, o losa de hormigón, más o menos armado, prefabricado y cuyas características son:

a) Para los elementos-lámina (verticales):

-dimensiones: entre 3 y $12 \mathrm{~m}$ de longitud; altura no mayor de $3 \mathrm{~m}$ y un espesor que oscila entre 6 y $24 \mathrm{~cm}$. -peso: máximo de hasta $10 \mathrm{Tm}$.

b) Para los elementos-losa (horizontales):

-dimensiones: ancho aproximado a $3 \mathrm{~m}$; luz no mayor de $5,50 \mathrm{~m}$; espesor 14 y $18 \mathrm{~cm}$. -peso máximo: de hasta $4 \mathrm{Tm}$.

Los elementos-lámina asumen las siguientes funciones:

-Resistencia a esfuerzos verticales

-Arriostramiento del edificio

-División con el exterior

-Divisiones interiores.

Las dos primeras pueden llevar, consigo o no, cualquiera de las dos últimas.

Los elementos-losa cumplen, por su lado:

-Resistencia a esfuerzos verticales.

- Arriostramiento del edificio

-División horizontal interior.

-División horizontal interior-exterior (caso de techo de última planta y suelo de primera planta)

Dichos elementos suelen disponer de:

-Terminación de sus paramentos, de tal forma que sólo precisan la incorporación en obra de los materiales de acabado (pavimentos, pinturas, papeles, etc.)

-Los elementos de fachada suelen salir de fábrica, con su terminación definitiva

- Aislamiento térmico, en caso necesario.

- Aislamiento acústico, dado por la compacidad del hormigón

-Incorporación de instalaciones secas (electricidad, telefonía, TV, FM, etc.).

-Incorporación de carpinterías: ventanas y cercos de puertas. 
Pueden ser:

-Homogéneos, de hormigón, los elementos interiores.

-Multicapa (de hormigón con interposición de una capa aislante térmica), los elementos de división interiorexterior.

\section{EL SISTEMA INDAGSA}

\subsection{Generalidades sobre el sistema}

El Sistema Constructivo INDAGSA se basa en la producción en taller, de forma racionalizada y con sistemas industriales, de paneles de hormigón armado y pretensado.

El Sistema Constructivo INDAGSA está enmarcado dentro del grupo de paneles de hormigón armado previstos para trasdosar.

Estos elementos, una vez montados en obra, constituirán la estructura y el cerramiento de todo edificio.

Los elementos que constituyen el Sistema INDAGSA, se agrupan en seis clases fundamentales, y de tal forma que el conjunto de estas seis clases, utilizadas simultáneamente en la realización de un edificio, definen el Sistema INDAGSA como un sistema de prefabricación completo.

Por otra parte, cada una de las clases de elementos del Sistema INDAGSA está concebida individualmente para poder combinarse con sistemas constructivos tradicionales prefabricados; consiguiendo, de esta forma, un sistema abierto.

\subsection{Descripción de los elementos del sistema}

\section{a) Elementos de clase I}

Paneles de hormigón armado, destinados a elementos interiores, portantes, autoportantes o de arriostramiento.

Estos paneles trabajan verticalmente, y estarán preparados para recibir forjados y prelosas prefabricadas del mismo taller, o forjados cerámicos tradicionalmente usados en construcción.

Se fabricarán en 10,12 ó $14 \mathrm{~cm}$ de espesor, alturas de 2,90 $\mathrm{m}$ a 3,50 $\mathrm{m}$ y longitud variable hasta un máximo de $10 \mathrm{~m}$.

Se sitúan, fundamentalmente, en separaciones de viviendas, cajas de ascensor y escalera.

\section{b) Elementos de clase II}

Paneles de hormigón armado destinados a fachadas portantes, autoportantes o de arriostramiento.
Trabajarán verticalmente y estarán preparados para recibir cualquier tipo de forjado que sea capaz de recibir la clase I de paneles.

Se fabricarán en 10,12 ó $14 \mathrm{~cm}$ de espesor, alturas de 2,90 a $3,50 \mathrm{~m}$ y de longitud variable, hasta un máximo de $10,00 \mathrm{~m}$.

Admiten terminaciones de hormigón visto, conseguidas a base de tratamientos de su cara externa, con ácido, retardadores, chorro de arena, etc. y formas superficiales diferentes, empleando útiles especiales y combinación de diferentes hormigones.

Están pensados para trasdosar con tabique de cartón-yeso o fábrica de ladrillo; admitiendo alternativas de fabricación de paneles sandwichs, para edificaciones industriales, fundamentalmente

\section{c) Elementos de Clase III}

Paneles de hormigón armado de 4 a $8 \mathrm{~cm}$ de espesor, destinados a fachadas ligeras y de hormigón arquitectónico.

Se utilizan combinados con los elementos de la clase II o entre sí, originando fachadas cortina.

Admite su utilización con estructuras tradicionales de hormigón armado, o metálicas.

Su longitud y anchura son variables, y pueden adoptar diferentes formas de borde y secciones según cumplan funciones de entrepaños, fachadas completas con huecos incorporados, antepechos, etc.

Estos paneles se caracterizan por no cumplir ninguna función estructural, resistente ni de arriostramiento.

El sistema de montaje (isostáticamente realizado), les permite libres dilataciones y retracciones bajo efectos térmicos $y$, en su dimensionamiento, se ha tenido en cuenta, exclusivamente, las cargas de fabricación, transporte y montaje.

\section{d) Elementos de Clase IV}

Paneles y prelosas de hormigón armado o pretensado, destinados a constituir los forjados (Fig. 1).

Son paneles simplemente nervados, con un intereje de $0,70 \mathrm{~m}$, ancho variable, hasta un máximo de $2,10 \mathrm{~m}$, canto variable entre 23 y $29 \mathrm{~cm}$.

Llevan previstos los pasos de conducción, tanto vertical como horizontalmente. 


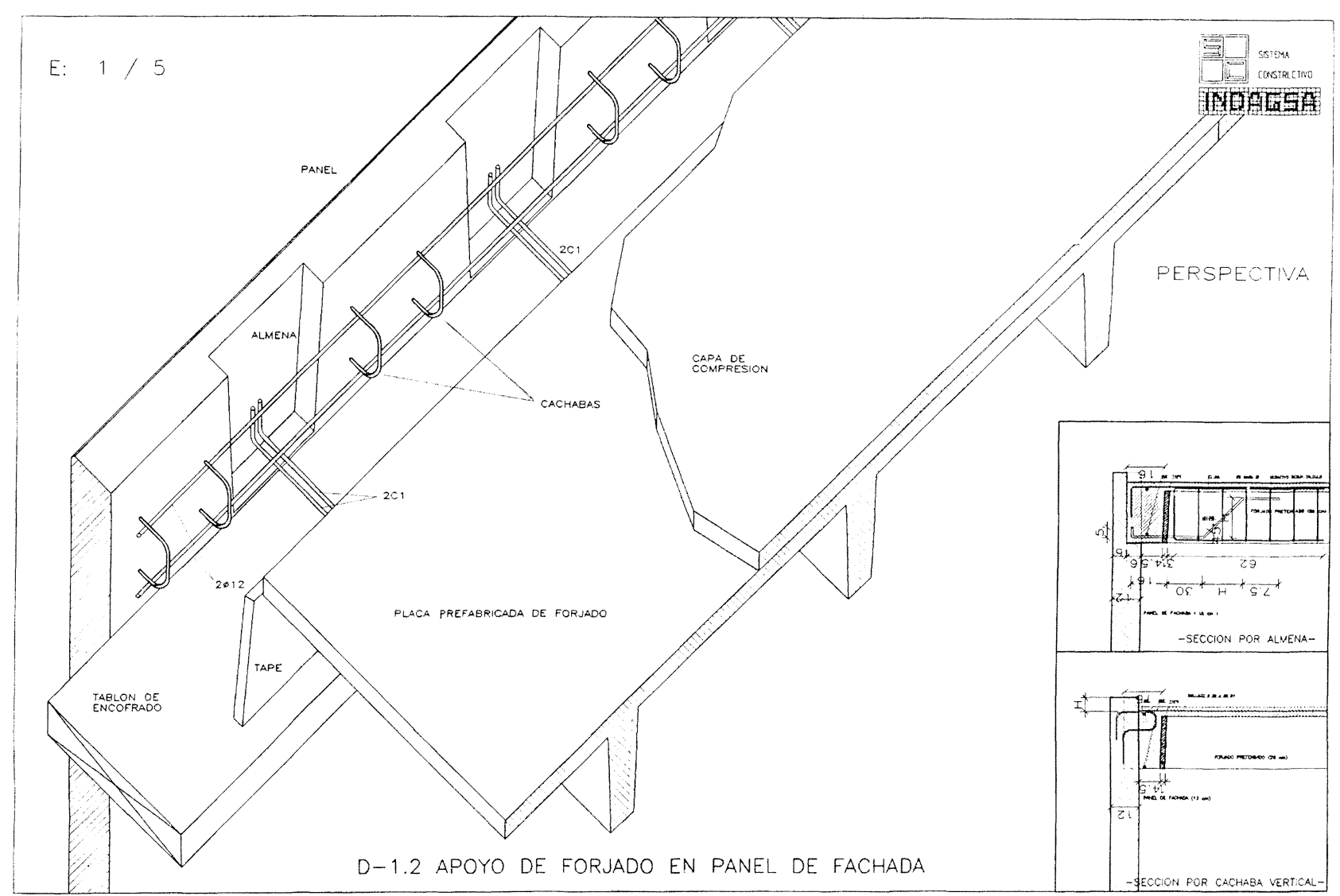

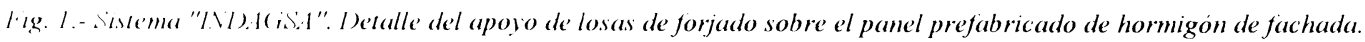

Son los elementos destinados a soportar las cargas verticales que se originan en cada tablero de piso, individualmente.

Cumplen también la función de transmitir y distribuir las cargas horizontales a los elementos de la clase I y II, destinados a dar rigidez transversal al edificio.

Admite la variante de prelosas armadas.

\section{e) Eilementos de (lase I}

Elementos de diseño lineal, vigas y dinteles, destinados a completar la función estructural de las clases I y II (Figs. 2, 3 y 4 ).

Se fabrican en 15 y $10 \mathrm{~cm}$, respectivamente, de espesor; cantos de 45 y $70 \mathrm{~cm}$ y longitudes variables, hasta un máximo de $8,0 \mathrm{~m}$.

\section{f)Elementos de (lase II}

Piezas especiales para rampas de escalera, paneles de instalaciones, losas de ascensor, etc.

Son piezas de geometría variable que resuelven los problemas particulares de cada obra

\subsection{Resumen de las características fundamentales}

\subsubsection{Características técnicas:}

-La producción en taller, permite tener un control de calidad propio de la industria, garantizando las calidades últimas del producto terminado.

-Se trata de un sistema abierto, que combina perfectamente con otras soluciones constructivas.

-Es de junta seca. Las uniones entre paneles se realizan con anclajes soldados, lo que confiere rigidez instantánea al edificio y proporciona una gran velocidad de montaje y ejecución de obra.

- Al estar previsto para trasdosar, las características internas del edificio son totalmente tradicionales, de ladrillo o yeso-cartón, no apareciendo, en ningún momento, hormigón en el interior de la vivienda.

-Esta misma solución de trasdosado permite una técnica de doble sellado externo-interno de las juntas, así como un perfecto tratamiento de los puentes térmicos y una ventilación de las cámaras; garantizando, de esta forma, la vivienda frente a humedades de origen externo y condensación. 

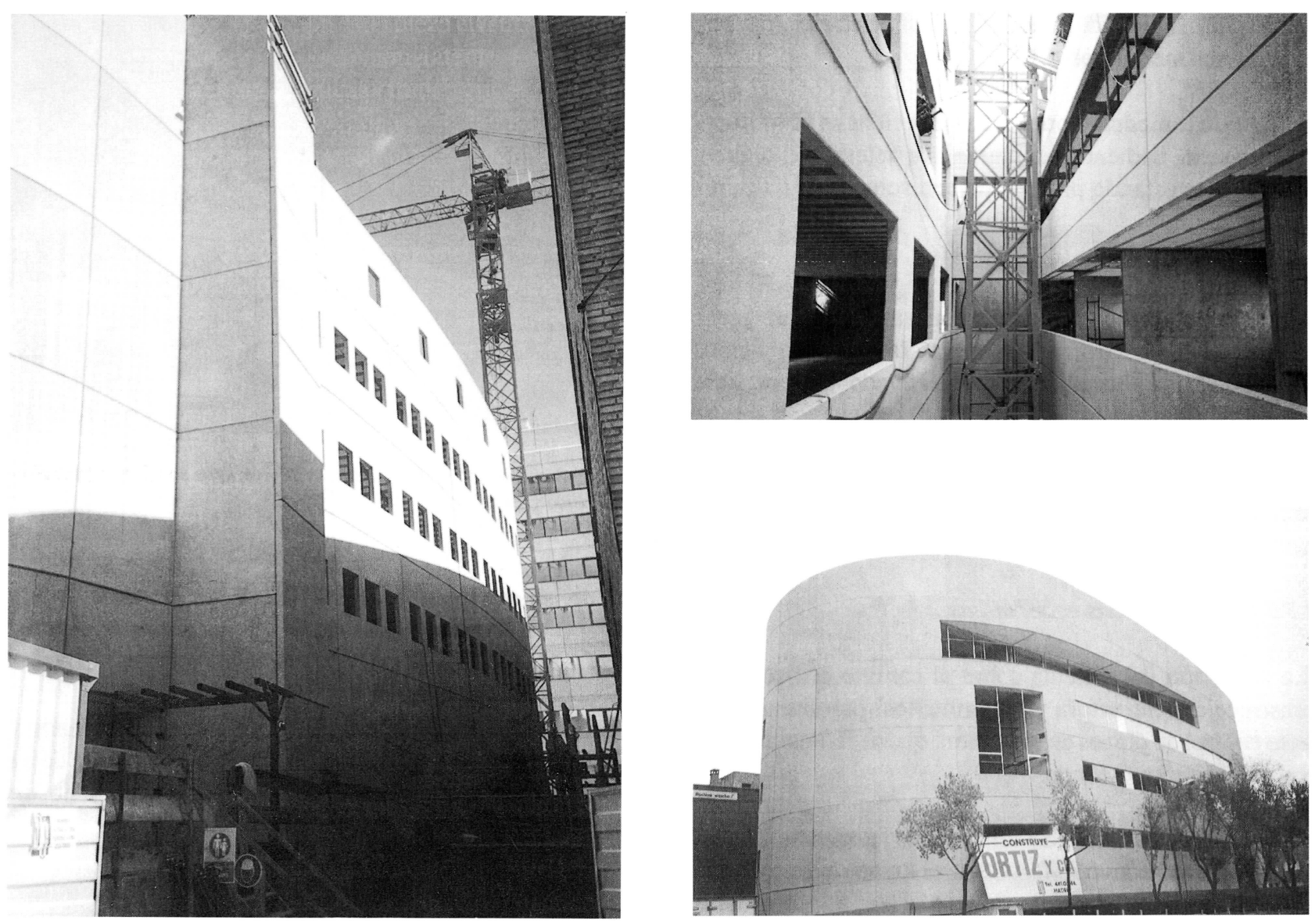

Figs. 2, 3 y 4.- Sistema "INDAGSA". Distintas fases de montaje de edificio industrial en Madrid (Arquitectos: Franco y Palao)

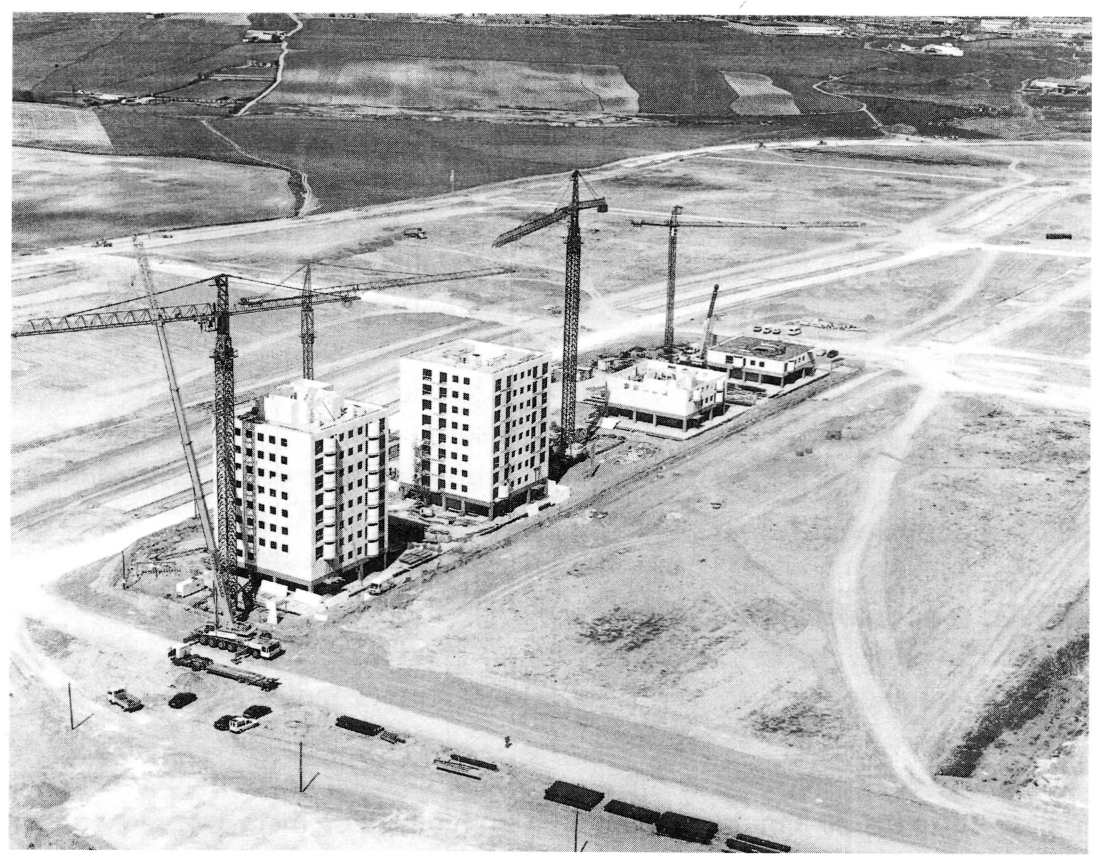

Fig. 5.- Sistema "INDAGSA". Aspecto de la fase de montaje de cuatro edificios de viviendas en Fuenlabrada-Madrid (Arquitecto: A. Perea)

-La solución de forjado nervado, complementando con falsos techos, nos permite la realización de instalaciones de trazado racional y fácilmente accesibles (Fig. 5).

(c) Consejo Superior de Investigaciones Científicas Licencia Creative Commons 3.0 España (by-nc)

\subsubsection{Características compositivas}

-El Sistema Constructivo INDAGSA, se basa en una http://informesdelaconstruccion.revistas.csic.es 
producción industrial, que no se ve condicionado por imperativos modulares.

- Al no existir modulaciones preestablecidas se tiene libertad de diseño, pudiendo adaptarse este sistema a cualquier edificio, aun estando proyectado con soluciones tradicionales.

-La calidad de las fachadas es de hormigón arquitectónico, hormigones de color con áridos naturales y tratamientos superficiales de chorro de arena, lavado con ácido, texturizado con elastómeros, o desactivado, de superficie vista (Fig. 6)

-La estructura es totalmente plana, tanto en su concepto de techos como de paredes, no apareciendo en ningún momento cuelgues de vigas ni mochetas de pilares.

\subsubsection{Características económicas}

-La actuación del sistema sobre el camino crítico de la construcción (estructura y cerramientos) permite un perfecto control de plazos de ejecución, que se reflejarán en el coste final de la vivienda.

-El peso de conjunto, de un edificio ejecutado con el Sistema Constructivo INDAGSA, es menor que con cualquier otro sistema prefabricado, lo que amplía, considerablemente, el radio de acción de la fábrica.

-La relación superficie útil/superficie edificada, es mayor que en los sistemas tradicionales y cualquier otro sistema de prefabricación.

-Por su tecnología, no modular, se pueden proyectar pequeñas unidades de obra, de forma más rentable.

\section{CONSTRUIR CON MÓDULOS TRIDIMENSIONALES}

\section{CONSIDERACIONES GENERALES}

\subsection{Concepto de módulo tridimensional}

Los módulos tridimensionales son componentes constructivos especiales de grandes dimensiones, que salen de fábrica totalmente acabados y equipados

Vienen a tener unas dimensiones no mayores a $3 \mathrm{mx}$ $3 \mathrm{~m} \times 10 \mathrm{~m}$, oscilando su peso entre las $20 \mathrm{Tm}$ que puede tener un módulo de hormigón y las $2 \mathrm{Tm}$ que alcanzan los módulos ligeros.

De lo que se trata en esta tecnología no es sólo de industrializar la obra gruesa, sino también de la incorporación de instalaciones, carpinterías y acabados, así como

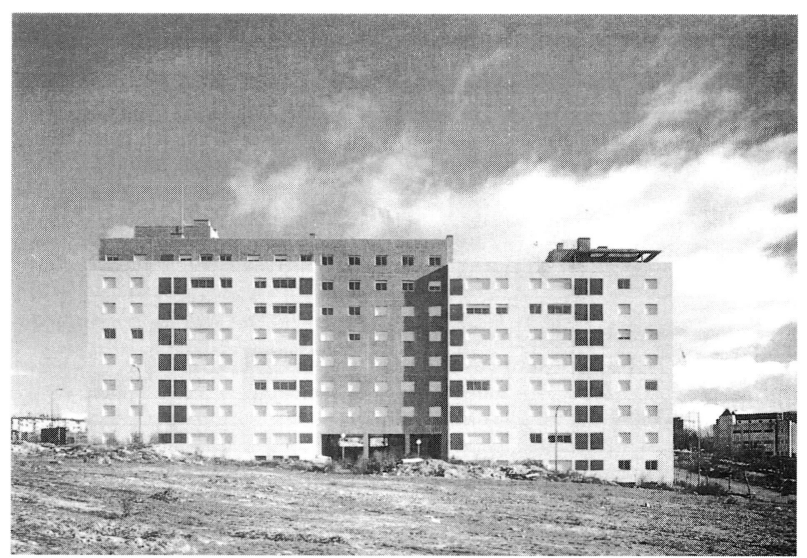

Fig. 6.- Sistema "INDAGSA". Edificio de viviendas en Madrid (Arquitecto: Franco y Palao).

las divisiones interiores y las superficies externas, dando lugar a verdaderos "contenedores", que quedarán "perdidos" cuando se coloquen en obra.

Con ello obtenemos un producto totalmente terminado y listo para su utilización en cualquier momento, fruto de un tratamiento científico del proceso productivo y de un elevado grado de mecanización.

Los sistemas con módulos tridimensionales permiten, como ningún otro procedimiento constructivo, acortar el tiempo de construcción, coordinando ejemplarmente las tareas del tajo, con las de fabricación.

\subsection{Clasificación}

Quizá la clasificación más decisoria sea la que se fija en el material que compone básicamente el módulo. Así:

-Módulos "pesados", son los fabricados, principalmente, con hormigón más o menos armado.

-Módulos "ligeros", todos los demás, en los que se busca la disminución de peso, mediante diversos procedimientos: la utilización de estructuras lineales metálicas, cáscaras de plástico o G.R.C. y uso de planchas de materiales ligeros (metacrilatos, contrachapados de madera, vidrio, chapas metálicas, PVC, etc.).

\subsection{Antecedentes históricos}

La aplicación de los módulos tridimensionales de hormigón comenzó a mediados de los años cincuenta, fundamentalmente formando bloques sanitarios que se incorporaban a los edificios construidos con grandes paneles, sobre todo, en la antigua URSS y en Polonia, donde se inició el uso para viviendas, dando lugar a la aparición de una maquinaria pesada importante para la fabricación, la manipulación y el transporte. 
Fuera de este entorno, en diversos países europeos, se comienza la edificación con estos módulos a final de los sesenta y principio de los setenta, pero con un nivel de calidad muy superior.

En EE.UU., y Japón también, se empezaron realizaciones en los años setenta, pero, salvo excepciones, como un hotel en San Antonio, Texas, la generalidad lo forman construcciones con módulos ligeros, que están perfeccionándose continuamente.

No obstante, la realización más famosa fue el célebre Habitat-67 de la Exposición Universal de Montreal, diseñado por Moshe Safdie y que constituyó para él un primer eslabón de una cadena de realizaciones a lo largo del mundo. El Habitat-67 se tuvo que construir con módulos de hormigón pretensado, debido a los muchos voladizos que precisaba el proyecto. Su costo se estimó en unas cuatro veces mayor a si se hubiera realizado con procedimientos tradicionales.

\subsection{Aspectos complementarios}

Hay que indicar que el mayor número de aplicaciones de módulos tridimensionales se está haciendo en viviendas unifamiliares, en las que muchas veces, sobre todo con módulos pesados, se producen casos de enmascaramiento o camuflaje para huir de la apariencia industrializada. En el caso de módulos ligeros, su desarrollo en este sector de vivienda unifamiliar, adopta, a veces, un carácter experimental, casi diríamos que "deslumbrador"; en otras ocasiones se precipitan hacia soluciones similares a las "mobile home" americanas.

Los módulos tridimensionales se utilizan, sobre todo, en edificios residenciales (viviendas, hoteles, etc.), edificios escolares, centros sanitarios primarios, construcciones administrativas básicas, etc. En países como EE.UU. o Japón, también se están extendiendo a edificios de oficinas y de apartamentos de altura.

Existe una utilización más modesta, pero que puede llegar a ser muy importante, que es la incorporación, en todo tipo de edificios, de módulos pesados especializados conteniendo escaleras, huecos de ascensores y bloques sanitarios, principalmente, como veremos seguidamente.

\section{EL SISTEMA "SET HOME"}

\subsection{Descripción del sistema}

El sistema SET HOME de construcción de viviendas, es un procedimiento que permite la industrialización de ciertos elementos del edificio, precisamente aquellos que constituyen la parte esencial del mismo: ESTRUCTURA e INSTALACIONES.
Se basa en la utilización de unos módulos tridimensionales construidos en hormigón armado que constituyen los recintos de COCINA, BAÑOS, ESCALERA y ASCENSOR, y que, colocados en cada planta del edificio, sirven de elementos de estructura vertical para soportar los forjados de piso de la planta superior.

Al mismo tiempo, dichos módulos contienen todas las instalaciones de las viviendas, tanto las interiores como las exteriores a los propios módulos, permitiendo, con ello, la normalización e industrialización de las mismas.

En las Figuras 7 y 8 se puede ver la distribución típica de un conjunto de viviendas construidas con los Módulos SET
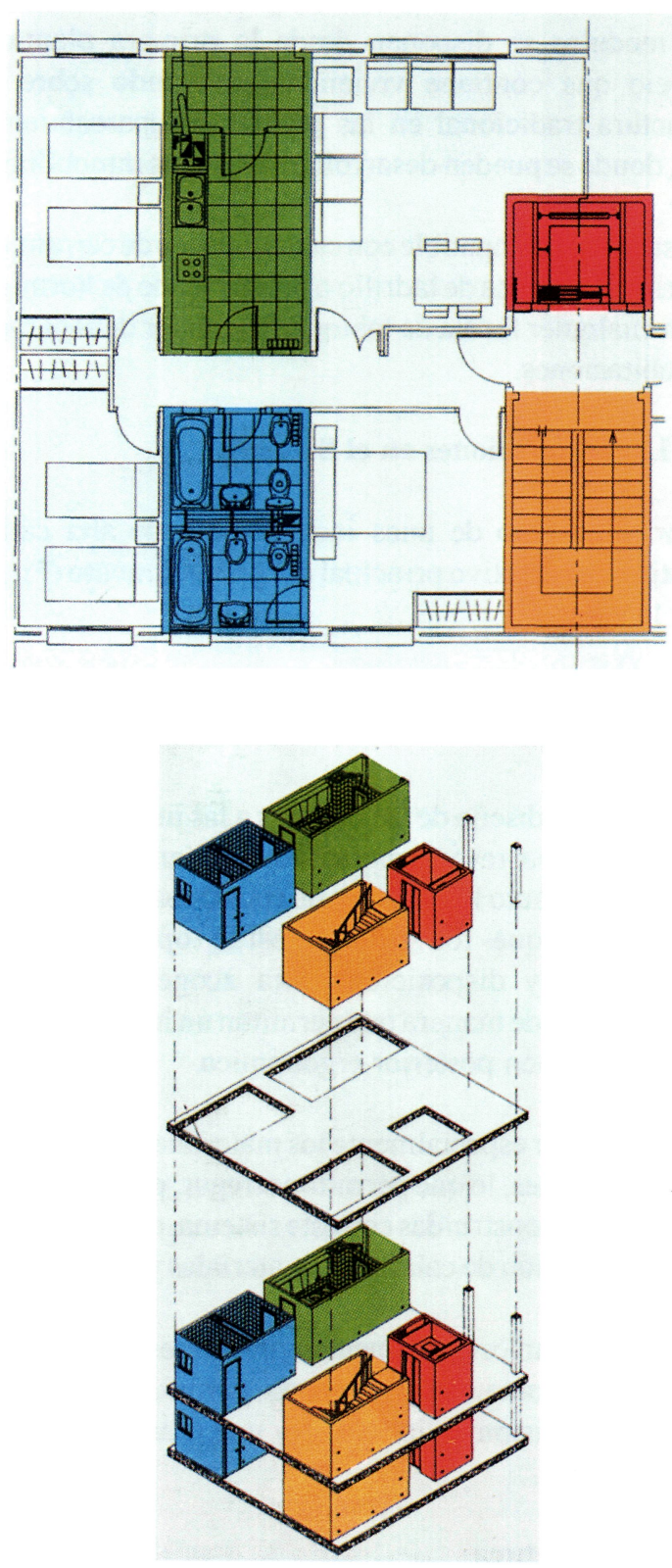

Figs. 7 y 8.- Sistema "SET HOME". Distribución de los módulos en un edificio de viviendas. 
HOME en el que, jugando con la disposición de dichos módulos, puede obtenerse cualquier distribución de viviendas

Dicha distribución permite observar las siguientes posibilidades del sistema:

a) La separación máxima entre dos Módulos permite la construcción de viviendas hasta un tamaño construido de $110 / 120 \mathrm{~m}^{2}$ con forjados tradicionales (luces de alrededor de $5 \mathrm{~m}$ ) y un número mínimo de pilares.

b) Cada recinto exterior a los módulos tiene, al menos, una pared de un Módulo, con lo que está servido por las instalaciones del mismo, pudiéndose evitar la existencia de instalaciones en el resto de la tabiquería.

Los módulos se disponen desde la primera planta del edificio que contiene viviendas, apoyando sobre una estructura tradicional en las plantas de aparcamiento y baja, donde se pueden desarrollar otros usos inmobiliarios.

El sistema, es compatible con cualquier tipo de cerramiento exterior, de fábrica de ladrillo o prefabricado de hormigón, y con cualquier forma de tabiquería interior de separación de habitaciones.

\subsection{Las instalaciones en el Sistema}

Dotar al edificio de unas instalaciones de alta calidad constituye el objetivo principal del procedimiento (Figs. 9, 10 y 11).

Para ello, el sistema de construcción se basa en tres criterios:

a) Adaptar el diseño de las fábricas a las instalaciones que contienen, y noal revés, como suele ocurrir en la construcción tradicional. Tanto los recintos de cocina, baños, etc., como las paredes que los forman están optimizados, en dimensiones y disposición, para acoger en ellos las instalaciones, de manera que permitan un montaje sencillo y una utilización posterior ergonómica.

b) Seleccionar especialmente los materiales empleados en las instalaciones, lo que permite entregar, posteriormente, las viviendas construidas con este sistema, con certificados de homologación de calidad de materiales y componentes.

c) Industrializar los componentes y conjuntos de los mismos, así como su montaje en la vivienda, permitiendo, con ello, una gran precisión en las tareas y una gran calidad en su producto final.

\subsection{La estructura}

Un edificio de viviendas construido con el Sistema se soporta a partir de un entramado estructural, formado por (c) Consejo Superior de Investigaciones Científicas
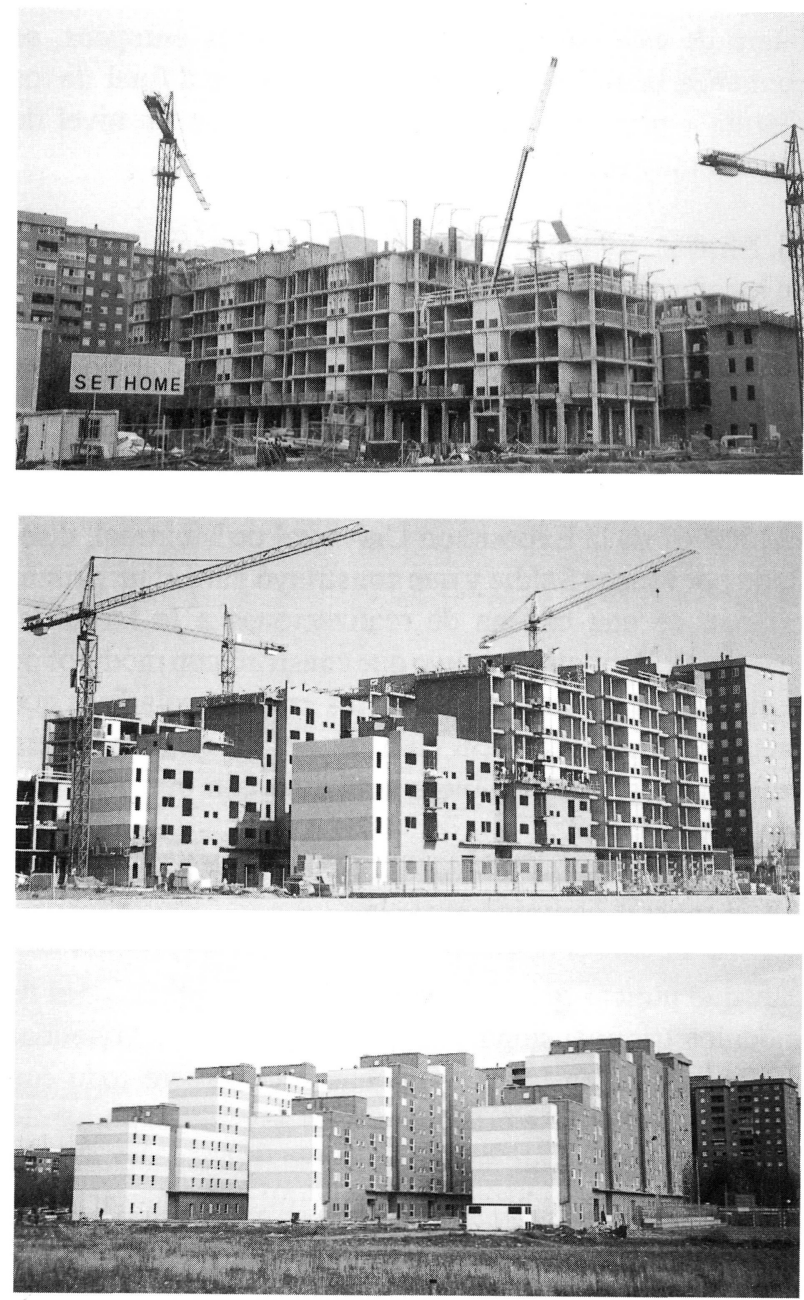

Figs. 9, 10 y 11.- Sistema "SET HOME". Distintas fases de montaje de un edificio de viviendas en Alcalá de Henares-Madrid(Arquitecto: E. Bardají).

los módulos tridimensionales descritos anteriormente y forjados planos de piso que apoyan sobre ellos.

Los módulos, construidos con hormigón armado, constituyen los elementos verticales de la estructura, proporcionando unagran rigidezy monolitismoal sistema. Sobre estos módulos apoyan los forjados de piso, constituidos por cualquiera de los procedimientos tradicionales, de viguetas y bovedillas, o de prelosas de hormigón armado, también prefabricadas, que constituyen, con una capa de hormigón continua sobre cada planta, el forjado horizontal de la estructura.

\subsection{El control y la reducción de tiempos}

El sistema conlleva una importante reducción en los plazos de construcción

Ello se logra mediante el solape de importantes actividades de obra, con lo que puede dividirse la construcción de un edificio de viviendas en tres etapas:

http://informesdelaconstruccion.revistas.csic.es 
I. Construcción, por procedimientos convencionales, de las plantas bajay de aparcamiento, y construcción simultánea en fábrica de la estructura del edificio, es decir, de los módulos y prelosas de forjado

II. Montaje en obra de la estructura del edificio, módulos y forjados, así como de la cubierta.

III. Construcción simultánea de los elementos que están dentro y fuera de los módulos.

\section{EL SISTEMA TRANSLOKO}

\subsection{Generalidades}

Es un sistema francés de construcción con módulos tridimensionales ligeros, realizados totalmente en fábrica. Desde allí se trasladan a cada obra, en donde, a través de unas operaciones muy sencillas de montaje y de conexión de instalaciones, se colocan en su posición definitiva (Fig. 12).

\subsection{Composición de los módulos}

a) Estructura del módulo: cada unidad es portante y está construida a base de un conjunto de perfiles en acero soldado indeformable, laminado en frío; cada módulo está formado por cuatro pilares, unidos por jácenas superiores oinferiores, con los nudos de unión arriostrados, haciéndolos rígidos. Vigas intermedias longitudinales y viguetas transversales, completan el conjunto.

b) Suelos: sobre las viguetas transversales de cada módulo, va una plancha de chapa galvanizada de $105 \mathrm{~mm}$ de sección grecada, que constituye el soporte resistente de cada suelo. Sobre esta plancha se colocan los distintos elementos de un sandwich, formado por un aislamiento de poliestireno expandido de clasificación M-1 y de espesor de $40 \mathrm{~mm}$, sobre él, un panel de conglomerado de madera y espesor de $30 \mathrm{~mm} \mathrm{y}$, por último, el pavimento final.

c) Techos: están también constituidos por un sandwich, formado por un panel melaminado de clasificación $\mathrm{M}-1$, espesor de $12 \mathrm{~mm}$ y color blanco satinado. Este panel está fijado a otro panel de yeso-cartón de $15 \mathrm{~mm}$; sobre éste, se coloca una capa de lana mineral rígida de espesor $40 \mathrm{~mm}$, sobre ella, un forro rígido que está suspendido mediante unos elementos de cuelgue y aún, sobre este forro, se extiende otra capa de lana mineral suelta.

d) Fachadas: están, asimismo, compuestas de una superposición de elementos. Así, desde el interior hacia el exterior, está compuesto de un panel melaminado de clasificación M-1, espesor de 12 mm y colorblanco satinado. A continuación, panel de yeso-cartón de $23 \mathrm{~mm}$, una capa de lana mineral rígida, con un espesor de $45 \mathrm{~mm}$, otra capa de lana mineral suelta de espesor $40 \mathrm{~mm}$ y una cha(c) Consejo Superior de Investigaciones Científicas

Licencia Creative Commons 3.0 España (by-nc)

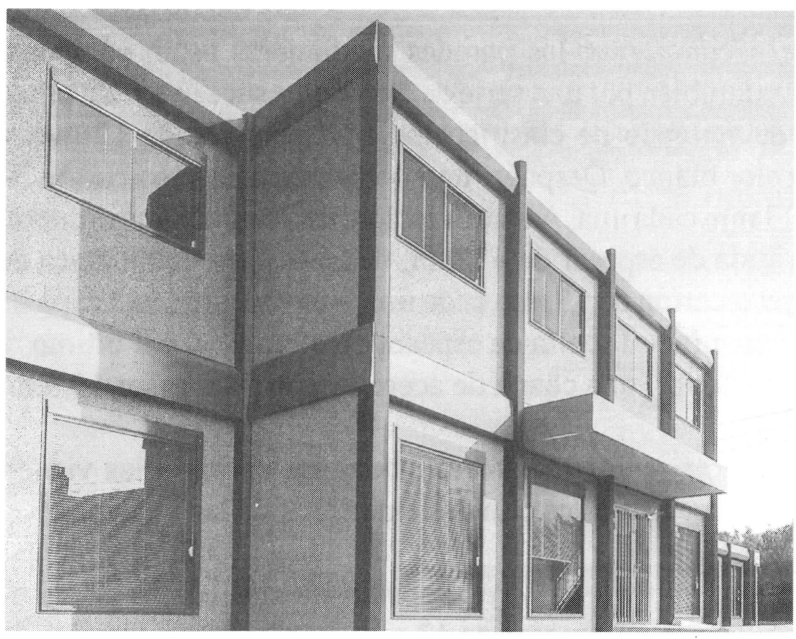

Fig. 12.- Sistema "TRANSLOKO". Ejemplo de edificio de oficinas en Francia.
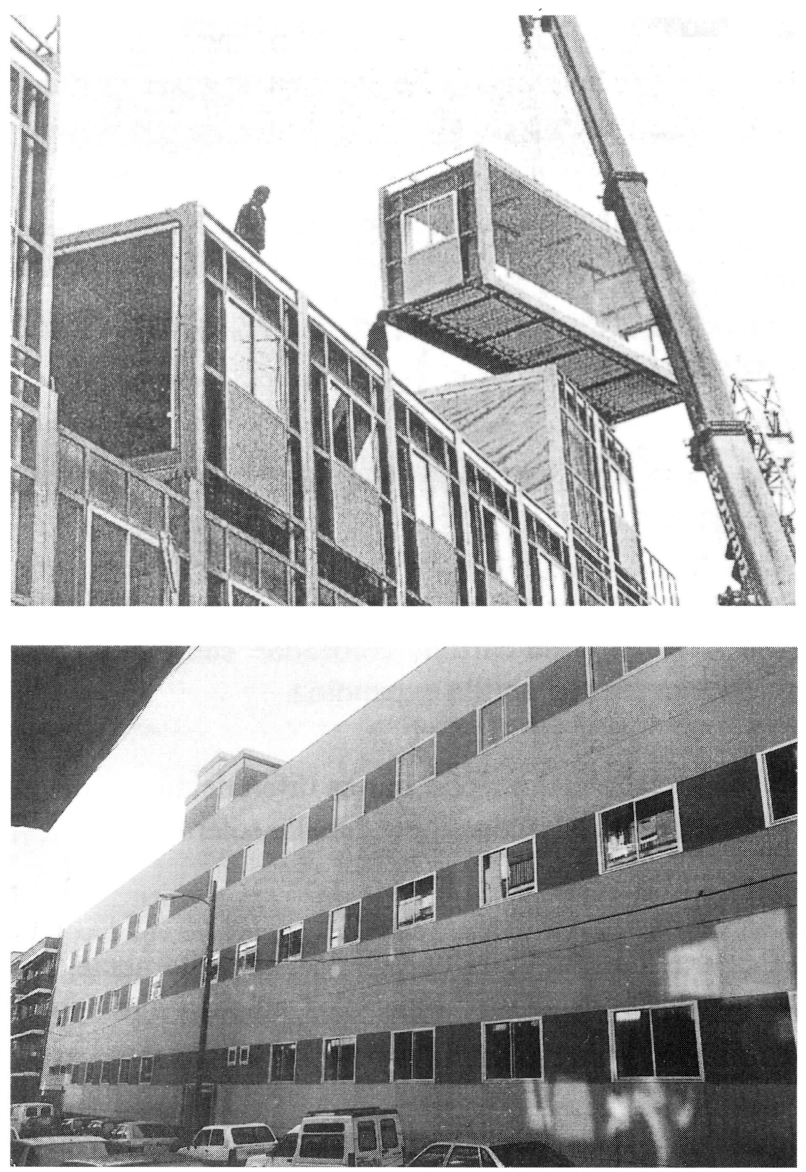

Figs. 13 y 14.- Sistema "TRANSLOKO". Edificio constituido por 72 módulos, con un total de más de $2.000 \mathrm{~m}^{2}$, destinado a laboratorios en Madrid (Arquitecto: P. Oliva).

pa galvanizada que sirve de soporte a este primer sandwich, de espesor $12 \mathrm{~mm}$. El resto del ancho del pilar, hasta llegar al exterior, queda como una cámara de aire vacía de unos $220 \mathrm{~mm}$, por último, se coloca el revestimiento exterior, que consiste en una chapa galvanizada precalada de 100 milésimas y espesor de $8 \mathrm{~mm}$, con un acabado gratinado, tipo revoco pétreo (Figs. 13 y 14). 
e) Medianerias: las paredes medianeras están compuestas también por una serie de elementos sucesivos. Un panel melaminado de clasificación $\mathrm{M}-1$, espesor de $12 \mathrm{~mm}$ y color blanco. Después, una doble placa de yeso-cartón de $13 \mathrm{~mm}$ cada una. A continuación, una capa de lana mineral rígida de espesor de $45 \mathrm{~mm}$, después, otra doble placa de yeso-cartón de $13 \mathrm{~mm}$ cada una, nuevamente, una capa de lana mineral suelta de espesor de $40 \mathrm{~mm}$ y, por último, y al exterior, una chapa de acero lacado de espesor $10 \mathrm{~mm}$.

f) Carpinterias exteriores: todas las carpinterias vienen incorporadas a los paneles exteriores de las fachadas.

g) Divisiones interiores: constan de dos placas de yesocartón, de un espesor de $13 \mathrm{~mm}$, con acabado exterior de melaminado blanco en las dos caras y una cámara interior de lana mineral, con un espesor total de $70 \mathrm{~mm}$. El conjunto de los paneles está anclado a techos y suelos mediante perfilería de aluminio anodizado, tanto en sentido vertical como horizontal y las juntas protegidas, mediante una omega y un tapajuntas.

\section{III. "ÚLTIMAS TENDENCIAS EN LA CONSTRUCCIÓN DE INTERIORES: LAS SOLERAS SECAS"}

\section{CONCEPTO DE SOLERA SECA: LA SOLERA "PLADUR"}

La solera seca es un suelo flotante, a base de placas especiales de yeso-cartón, colocadas sobre una base niveladora seca de arcilla expandida.

Se comercializan dos tipos, que se diferencian solamente por la inclusión o no de aislante. Son las soleras "aislantes" o "normales"

La solera "normal" está constituida, esencialmente, por dos placas de yeso-cartón especiales, colocadas sobre una base niveladora de arcilla expandida seca.

La solera "aislante" lleva incorporada a la placa inferior de yeso-cartón especial una plancha de poliestireno expandido.

\section{PROCESO DE EJECUCIÓN}

La ejecución es similar, tanto si se trata de la solera "normal" como de la "aislante".

\subsection{Limpieza y preparación de la capa de compresión} del forjado (Fig. 15)

Es preciso regularizar la superficie lo máximo posible y evitar que queden restos de yeso.

(c) Consejo Superior de Investigaciones Científicas Licencia Creative Commons 3.0 España (by-nc)

\subsection{Colocación de la junta perimetral (Fig. 16)}

Se situará una tira de fibra de vidrio, de $2 \mathrm{~cm}$ de espesor, a lo largo de todo el perímetro.

La altura será variable ,según el espesor de la solera.

\subsection{Trazado del nivel de la base niveladora en la junta perimetral}

\subsection{Colocación de lámina protectora (Fig. 17)}

Se situará una lámina especial de poliestireno sobre toda la zona a solar, rebasando la junta perimetral.
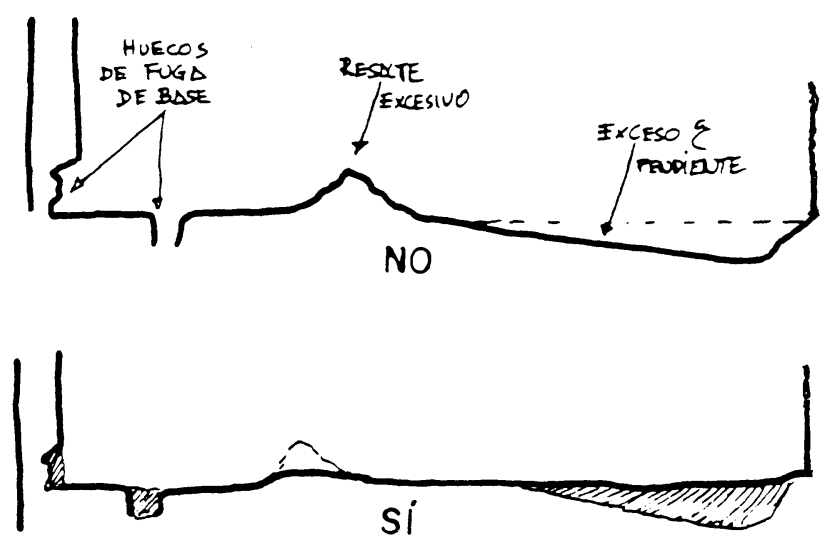

Fig. 15.- Soleras secas "PLADUR". Limpieza y preparación de la zona a solar.

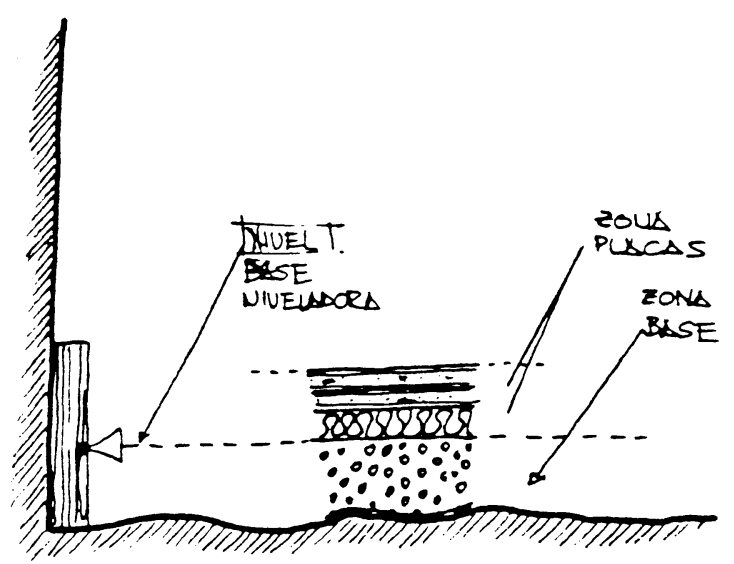

Fig. 16.- Colocación de junta perimetral y fijación de nivel.

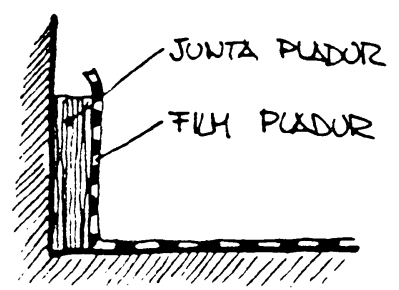

Fig. 17.- Colocación de lámina protectora.

http://informesdelaconstruccion.revistas.csic.es 


\subsection{Vertido y nivelación de la base niveladora (Fig. 18)}

Se realizará gracias a la disposición de reglas niveladoras, en zonas no mayores de 2,50 $\mathrm{m}$ de ancho y largos variables.

Se utiliza arcilla expandida para rellenos de espesores mayores de $2 \mathrm{~cm}$, o "arena", del mismo tipo de material que la base, para espesores menores.

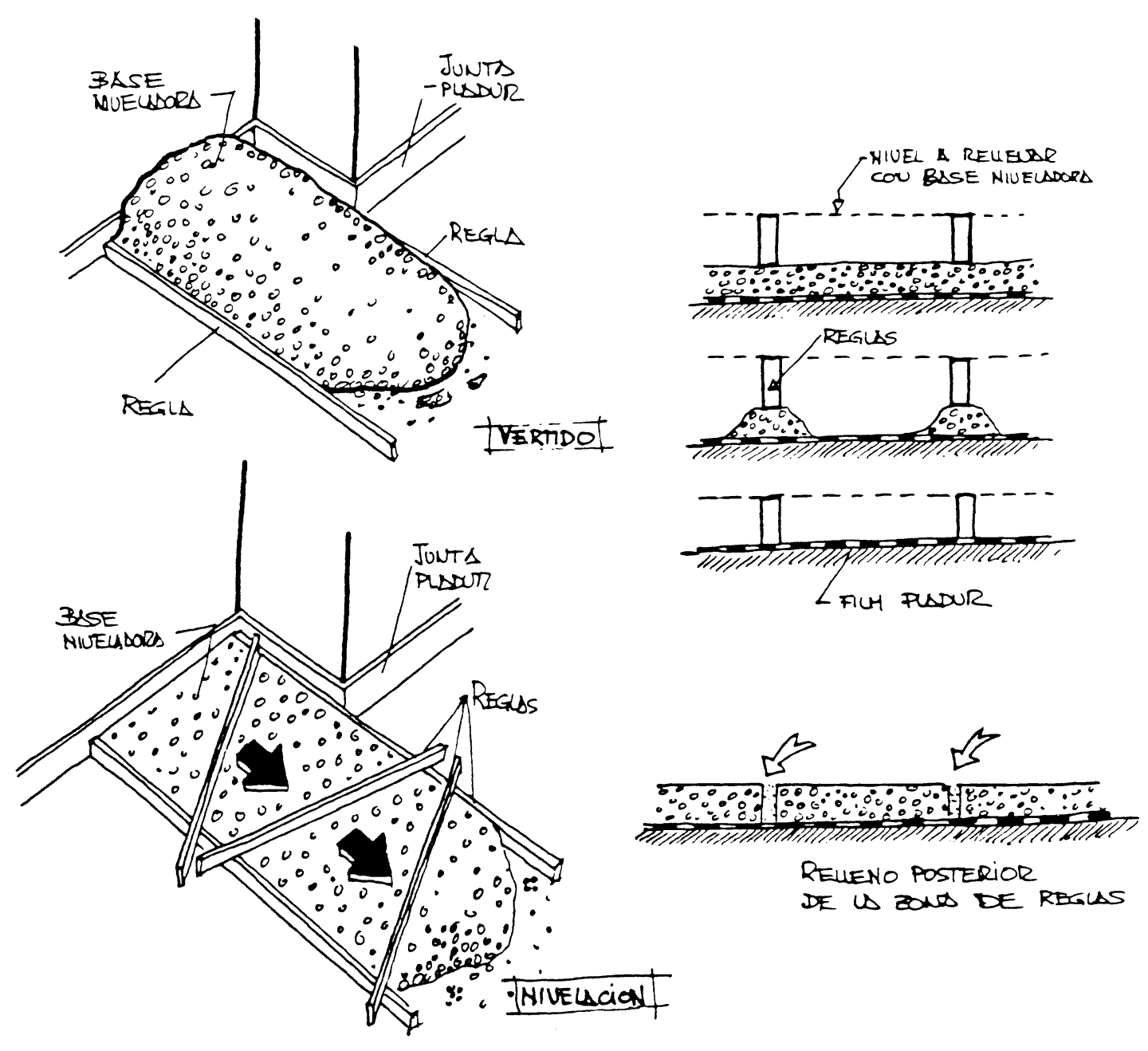

Fig. 18.- Vertido y extensión de base niveladora.

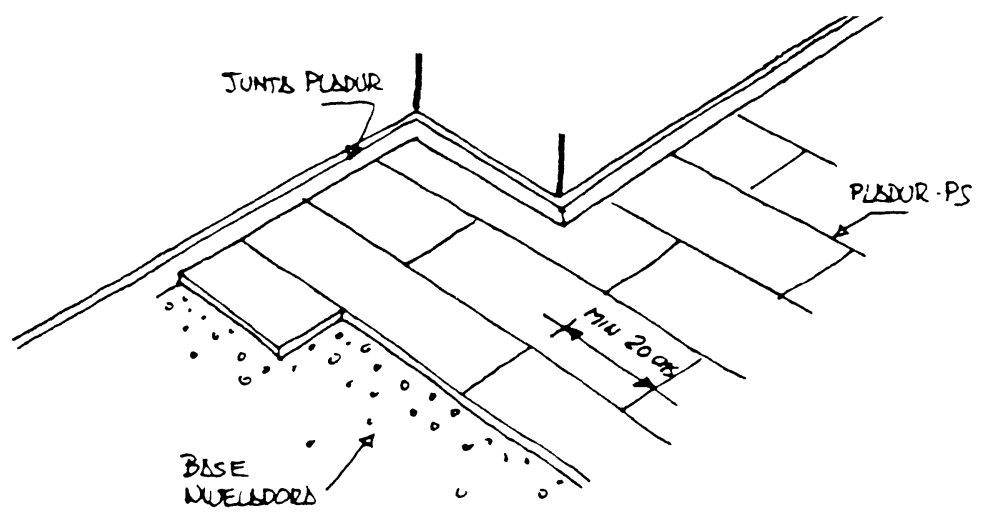

Fig. 19.- Colocación de $1^{a}$ capa de yeso-cartón especial.
2.6. Colocación de la primera placa de yeso-cartón (Fig. 19)

Esta primera placa puede llevar la plancha de poliestireno o no, según sea la solera "aislante" o "normal".

Se colocarán las placas directamente sobre la base niveladora, a "matajuntas" y "a tope". 
2.7. Colocación de la segunda placa de yeso-cartón (Fig. 20)

Se fijarán a la capa anterior mediante una cola especial.

Siempre se colocarán a "matajuntas"entre ellas. Se evitará, también, su coincidencia con las de la primera capa.

\subsection{Saneado de junta perimetral (Fig. 21)}

Transcurrido el tiempo de secado se saneará la junta perimetral, cortando la junta y la lámina protectora sobrantes.

\subsection{Imprimación}

Posteriormente, es importante realizar una imprimación de tipo vinílico sobre toda la superficie a solar, recomendándose, en zonas húmedas, dar dos manos de imprimación..

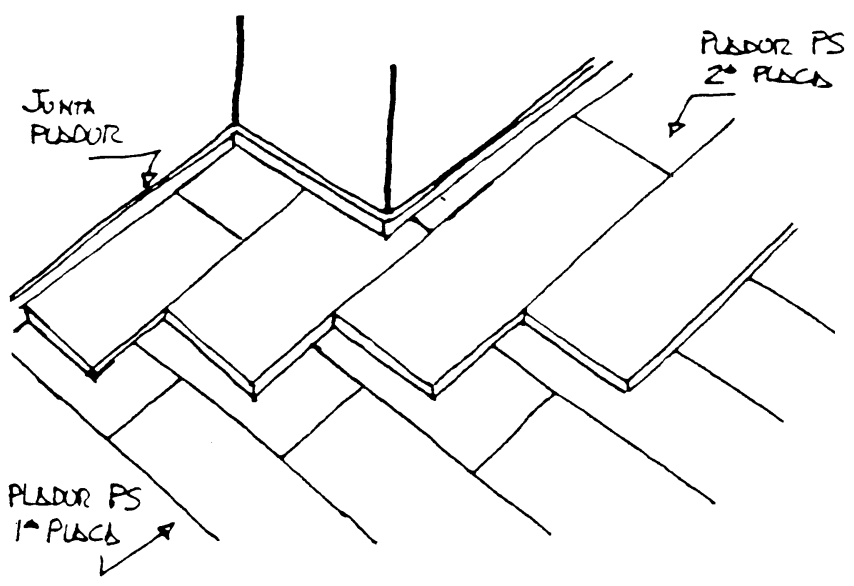

Fig. 20.- Colocación de segunda capa de yeso-cartón especial a "matajuntas" con la primera.

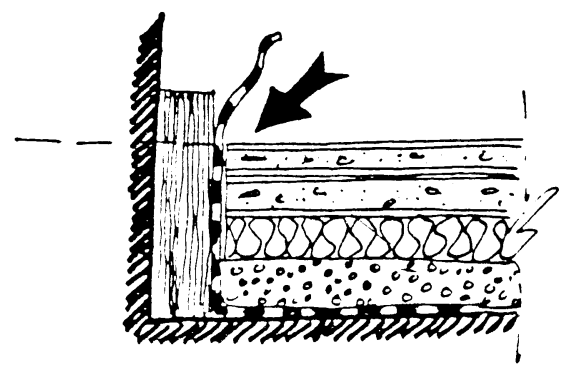

Fig. 21. - Corte de junta perimetral y de lámina protectora sobrantes. La solera seca está lista para recibir el pavimento.

\subsection{Solado}

Se pueden realizar todo tipo de pavimentos (moquetas, parquets, pavimentos sintéticos, baldosas de gres, etc).

Se guardarán las siguientes precauciones:

- Imprimación previa

- Mantenimiento limpio de la solera.

- Elección correcta del material de agarre o adhesivo, que sea compatible con la solera y el pavimento.

- Realizar las correctas juntas de dilatación según el tipo de pavimento.

- Mantener junta de dilatación del solado a lo largo de todo el perímetro.

\subsection{Divisiones verticales}

Sobre la solera seca no deben ejecutarse tabiques de albañilería y ejecución húmeda, bien sean de distribución, de separación de locales, o bien trasdosados.

En ese caso se realizarán antes de la ejecución de la solera seca.

Encima de la solera seca sólo se podrán colocar tabiques ligeros y de ejecución en seco, como son los de yeso-cartón, los paneles suelo-techo de yeso o escayola o los tabiquesmampara

\section{POSIBILIDADES DE APLICACIÓN}

-Las soleras secas son idóneas para edificación nueva $\mathrm{y}$, también, para la rehabilitación, por su poco peso ( sobre $\left.26 \mathrm{~kg} / \mathrm{m}^{2}\right)$.

-Son de ejecución rápida y sin aditivos húmedos. -Su colocación es independiente de las condiciones atmosféricas ambientales.

-Con escombros mínimos y, por tanto, con mayor limpieza que en la construccion tradicional.

-Mejora el aislamiento térmico del local

- Actúa como suelo flotante, ya que está desolidarizada del resto de los elementos por medio de la base niveladora y de la junta perimetral, por lo que disminuye la transmisión acústica por impacto, lo que hace muy apropiada sobre forjados monolíticos de hormigón

\section{AGRADECIMIENTOS}

Por último, quiero mostrar mi agradecimiento, por su colaboración, a los Servicios Técnicos de: INDAGSA, SET HOME; ARGO-TRANSLOKO y EPYSA-PLADUR. 